MATHEMATICS OF COMPUTATION

Volume 69, Number 230, Pages 867-879

S $0025-5718(99) 01127-8$

Article electronically published on May 17, 1999

\title{
THE THIRD LARGEST PRIME DIVISOR OF AN ODD PERFECT NUMBER EXCEEDS ONE HUNDRED
}

\author{
DOUGLAS E. IANNUCCI
}

\begin{abstract}
Let $\sigma(n)$ denote the sum of positive divisors of the natural number $n$. Such a number is said to be perfect if $\sigma(n)=2 n$. It is well known that a number is even and perfect if and only if it has the form $2^{p-1}\left(2^{p}-1\right)$ where $2^{p}-1$ is prime.

It is unknown whether or not odd perfect numbers exist, although many conditions necessary for their existence have been found. For example, Cohen and Hagis have shown that the largest prime divisor of an odd perfect number must exceed $10^{6}$, and Iannucci showed that the second largest must exceed $10^{4}$. In this paper, we prove that the third largest prime divisor of an odd perfect number must exceed 100.
\end{abstract}

\section{INTRODUCTION}

For the natural $n$, we denote the sum of its positive divisors by

$$
\sigma(n)=\sum_{d \mid n} d
$$

We define $n$ to be perfect if $\sigma(n)=2 n$.

An even number is perfect if and only if it has the form $2^{p-1}\left(2^{p}-1\right)$, where $2^{p}-1$ is prime.

Whether or not any odd perfect numbers exist is still unknown. Many conditions necessary for their existence have been found. We refer the reader to the introduction section of [5], where the author, in a brief history, mentions some of these results.

Let us consider the possible class of theorems for the existence of odd perfect numbers, given by

$\mathcal{P}(K, M)$. An odd perfect number is divisible by $K$ distinct primes, each of which exceed $M$.

The best result to date for the case $K=1$ was obtained by Cohen and Hagis [2]; namely, $\mathcal{P}\left(1,10^{6}\right)$. For $K=2$, we have the result of the author [5], $\mathcal{P}\left(2,10^{4}\right)$. We combine these results and state

Received by the editor December 12, 1997 and, in revised form, January 26, 1998 and June 2, 1998.

1991 Mathematics Subject Classification. Primary 11A25, 11Y70.

Key words and phrases. Perfect numbers, cyclotomic polynomials.

This paper presents the main result of the author's doctoral dissertation completed at Temple University in 1995 under the direction of Peter Hagis, Jr. 
Lemma 1. The largest prime divisor $P$ of an odd perfect exceeds $10^{6}$. The second largest prime divisor $S$ of an odd perfect number exceeds $10^{4}$.

The purpose of this paper is to obtain a theorem of the form $\mathcal{P}(3, M)$. In particular, we shall prove $\mathcal{P}\left(3,10^{2}\right)$, and we state this result as

Theorem 1. If $T$ is the third largest prime divisor of an odd perfect number, then $T>10^{2}$.

For an integer $k \geq 3$, we say that a natural number $n$ is multiply perfect with index $k$ (or, simply, $k$-perfect) if $\sigma(n)=k n$. Hagis [4] showed for all $k \geq 3$ that the third largest prime divisor of an odd $k$-perfect number exceeds $10^{2}$. Theorem 1 is the analogous statement of this result for the case when $k=2$. While there are some similarities between Hagis's proof and that of Theorem 1 the latter proof is rendered far more complicated by the smaller index of $k=2$.

Computation plays a huge role in the proof of Theorem [1. All computations and computer searches for this paper were conducted on an IBM-486 personal computer using a UBASIC software package. Verification of all primes was carried out using the APR primality test, due to Adleman, Pomerance and Rumely [1].

\section{SOME PRELIMINARIES}

Throughout this paper, nonnegative integers are denoted by $a, b, c, d, \alpha, \beta, \gamma$, as well as by $h, i, j, k, l, m, n$, and $H, I, J, K, L, M, N$. Primes, which are odd unless noted otherwise, are denoted by $\pi, p, q, r, s, t, u, v$, and $P, Q, R, S, T$.

We say $p^{k} \| m$ if $p^{k} \mid m$ but $p^{k+1} \nmid m$, and we say $v_{p}(m)=k$ if $p^{k} \| m$. If $p \nmid a$, we denote by $o_{p}(a)$ the exponent to which $a$ belongs, modulo $p$.

Recalling $\Phi_{m}(a)$ (i.e., the cyclotomic polynomial of order $m$ evaluated at $a$ ), we have

$$
b^{n}-1=\prod_{d \mid n} \Phi_{d}(b)
$$

and

$$
\sigma\left(p^{a}\right)=\prod_{\substack{d \mid a+1 \\ d>1}} \Phi_{d}(p) .
$$

If $N$ is an odd perfect number with unique prime factorization given by $\prod_{i=1}^{k} p_{i}^{a_{i}}$, we have

$$
2 N=\prod_{i=1}^{k} \prod_{\substack{d \mid a_{i}+1 \\ d>1}} \Phi_{d}\left(p_{i}\right) .
$$

We conclude this section by giving several results which will be applied frequently throughout this paper. We refer the reader to the preliminaries section of [5], where we gave these same results along with their references (or with brief proofs).

Lemma 2. For primes $p$ and $q, q \mid \Phi_{m}(p)$ if and only if $m=h q^{\gamma}$, where $h=o_{q}(p)$ and $\gamma \geq 0$. If $\gamma>0$, then $q \| \Phi_{m}(p)$. 
An immediate consequence of (2) and Lemma 2 is

$$
v_{q}\left(\sigma\left(p^{a}\right)\right)= \begin{cases}v_{q}\left(\Phi_{h}(p)\right)+v_{q}(a+1), & \text { if } h \mid a+1, h>1, \\ v_{q}(a+1), & \text { if } h=1, \\ 0, & \text { otherwise. }\end{cases}
$$

Lemma 3. If $q \mid \Phi_{a}(p)$ and $r \mid \Phi_{b}(p)$, where $a \neq b, q \equiv 1(\bmod a)$, and $r \equiv 1(\bmod b)$, then $q \neq r$.

Lemma 4. If $m \geq 3$, then $\Phi_{m}(p)$ has a prime divisor $q$ with the property $q \equiv 1$ $(\bmod m)$.

Lemma 5. An odd perfect number must have unique prime factorization given by

$$
\pi^{4 m+1} p_{1}^{2 a_{1}} p_{2}^{2 a_{2}} \cdots p_{k}^{2 a_{k}}
$$

where $\pi \equiv 1(\bmod 4)$.

We will refer to $\pi$ as the special prime. Recalling the function $\sigma_{-1}(n)=\sigma(n) / n$, we see that $n$ is perfect if and only if $\sigma_{-1}(n)=2$. The function $\sigma_{-1}$ is multiplicative, and

$$
\begin{gathered}
\sigma_{-1}\left(p^{a}\right)<\sigma_{-1}\left(p^{b}\right)<\frac{p}{p-1} \text { if } a<b \\
\sigma_{-1}\left(q^{b}\right)<\sigma_{-1}\left(p^{a}\right), \quad \text { for all } a>0 \text { and } b \geq 0, \text { if } p<q .
\end{gathered}
$$

\section{ACCEPtABle AND ADMissible POSItive INTEGERS}

The proof of Theorem 1 will be given in Sections 4, 5 and 6 In order to make the proof more tractable, it is necessary to provide two definitions. First, we consider the set of primes given by

$$
X=\{3,5,7,11,13,19,31,61,97\} .
$$

Next, we give

Definition 1. For $p \in X$ and $q<100$, let $h=o_{p}(q)$. We say that the positive integer $k$ is $(p, q)$-acceptable if each of the following is true:

1. $k+1=h p^{\gamma}$, where $\gamma \geq 0$.

2. $4 \nmid h$, and $2 \nmid h$ if $q \equiv 3(\bmod 4)$.

3. $\sigma\left(q^{k}\right)$ has no prime factor between $10^{2}$ and $10^{4}$.

4. $\sigma\left(q^{k}\right)$ has at most one prime factor between $10^{4}$ and $10^{6}$.

5. $\sigma\left(q^{k}\right)$ has at most two prime factors greater than $10^{4}$.

Let $\beta$ be the smallest positive integer such that $h p^{\beta} \geq 50$. It follows by Lemmata 4 and 3 , equation (2), and conditions 3, 4, and 5 of Definition 1 that $k$ is not $(p, q)$ acceptable if $k+1=h p^{\gamma}$ and $\gamma \geq \beta+2$. Thus the set of $(p, q)$-acceptable integers is finite.

TABle 1. The values $A_{p}, B_{p}, C_{p}$ and $\lambda(p)$ for $p \in X$

\begin{tabular}{|lllll|ccccc|ccccc|}
\hline$p$ & $A_{p}$ & $B_{p}$ & $C_{p}$ & $\lambda(p)$ & $p$ & $A_{p}$ & $B_{p}$ & $C_{p}$ & $\lambda(p)$ & $p$ & $A_{p}$ & $B_{p}$ & $C_{p}$ & $\lambda(p)$ \\
\hline 3 & 6 & 3 & 2 & 11 & 11 & 3 & 0 & 2 & 5 & 31 & 2 & 1 & 1 & 4 \\
5 & 0 & 1 & 1 & 2 & 13 & 3 & 0 & 1 & 4 & 61 & 2 & 0 & 0 & 2 \\
7 & 7 & 1 & 2 & 10 & 19 & 2 & 1 & 2 & 5 & 97 & 1 & 0 & 0 & 1 \\
\hline
\end{tabular}


A computer search was conducted and a list of all $(p, q)$-acceptable values was compiled. Then, for each fixed $p \in X$, four integers, each depending on $p$, were determined as follows:

1. $A_{p}$ denotes the sum $\sum v_{p}\left(\sigma\left(q^{k}\right)\right)$, taken over all $k$ and $q$ such that $k$ is even and $(p, q)$-acceptable, and such that $r<100$ if $r \mid \sigma\left(q^{k}\right)$.

2. $B_{p}$ denotes the maximum value of $v_{p}\left(\sigma\left(q^{k}\right)\right)$, taken over all $k$ and $q$ such that $k$ is odd and $(p, q)$-acceptable.

3. $C_{p}$ denotes the maximum value of all sums of the form $\sum v_{p}\left(\sigma\left(q^{k}\right)\right)$, where each sum is taken over $k$ and $q$ such that $k$ is even and $(p, q)$-acceptable, and such that $\sigma\left(q^{k}\right)$ is divisible by a prime exceeding $10^{4}$, but neither three distinct primes exceeding $10^{4}$ nor two distinct primes between $10^{4}$ and $10^{6}$ appear among the prime divisors of all values $\sigma\left(q^{k}\right)$ involved in the sum.

4. $\lambda(p)$ denotes the sum $A_{p}+B_{p}+C_{p}$.

The values $A_{p}, B_{p}, C_{p}$ and $\lambda(p)$ for all $p \in X$ are given in Table 1

Definition 2. If $p<100$ or if $p>10^{4}$, we say that the positive integer $d$ is $p$-admissible if each of the following is true:

1. $d$ is odd unless $p \equiv 1(\bmod 4)$, in which case $4 \nmid d$.

2. $\Phi_{d}(p)$ has no prime factor between $10^{2}$ and $10^{4}$.

3. $\Phi_{d}(p)$ has at most one prime factor between $10^{4}$ and $10^{6}$.

4. $\Phi_{d}(p)$ has at most two prime factors greater than $10^{4}$.

5. If $10^{4}<p<10^{6}$, then $\Phi_{d}(p)$ has no prime factors between $10^{4}$ and $10^{6}$, and at most one prime factor greater than $10^{6}$.

6. If $p>10^{6}$, then $\Phi_{d}(p)$ has at most one prime factor greater than $10^{4}$.

\section{Restrictions on $v_{p}(N)$ FOR $p \in X$}

We may now begin the proof of Theorem[, which is given by reductio ad absurdum. Without further explicit mention, we will assume that $N$ is an odd perfect number, all of whose distinct prime divisors, save two, are less than 100 . Let $P$ and $S$ denote the largest and second largest prime divisors of $N$, respectively, and let $\alpha=v_{P}(N), \beta=v_{S}(N)$. Then

$$
N=L S^{\beta} P^{\alpha},
$$

where $p<100$ if $p \mid L$. By Lemma 1 we have $S>10^{4}$ and $P>10^{6}$. Since $N$ is perfect, we have

$$
2 N=\sigma(L) \sigma\left(S^{\beta}\right) \sigma\left(P^{\alpha}\right) .
$$

Under these assumptions, we can find restrictions for the values $v_{p}(N)$ for $p \in X$. The remainder of this section is devoted to this end.

Suppose $p^{a} \| N$ and $d \mid a+1, d>1$. By (3), (7) and Lemma 1 it follows that conditions 2, 3, and 4 of Definition 2 must be satisfied by $d$ (as must conditions 5 and 6 if $p>10^{4}$ ). Furthermore, by Lemma [5, condition 1 of Definition 2 is also satisfied. We have proved

Lemma 6. If $d$ is not $p$-admissible, then $d \nmid v_{p}(N)+1$.

We now establish an upper bound on $v_{p}(\sigma(L))$ for $p \in X$.

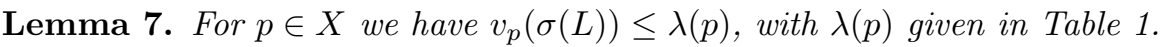


Proof. Suppose $q^{a} \| L$ and $p \mid \sigma\left(q^{a}\right)$. Let $h=o_{p}(q)$. Then $h \mid a+1$ by (4), so we may write $a+1=b h p^{\gamma}$, where $p \nmid b$ and $\gamma \geq 0$. Thus by (41) we have $v_{p}\left(\sigma\left(q^{a}\right)\right)=$ $v_{p}\left(\sigma\left(q^{k}\right)\right)$, where $k+1=h p^{\gamma}$. Since $k+1 \mid a+1, k$ satisfies condition 2 of Definition 1 by Lemma 5. Conditions 3,4 , and 5 are also satisfied by $k$, since $\sigma\left(q^{k}\right) \mid \sigma\left(q^{a}\right)$ and $\sigma\left(q^{a}\right) \mid 2 N$. Hence $k$ is $(p, q)$-acceptable.

Thus, if $L$ has unique prime factorization $\prod_{i=1}^{c} q_{i}^{a_{i}}$, and if $p \mid \sigma\left(q_{i}^{a_{i}}\right)$, there exists a $\left(p, q_{i}\right)$-acceptable value $k_{i}$ such that $v_{p}\left(\sigma\left(q_{i}^{a_{i}}\right)\right)=v_{p}\left(\sigma\left(q_{i}^{k_{i}}\right)\right)$. We may write

$$
v_{p}(\sigma(L))=\sum_{i=1}^{c} v_{p}\left(\sigma\left(q_{i}^{a_{i}}\right)\right)=\sum v_{p}\left(\sigma\left(q_{i}^{k_{i}}\right)\right)
$$

where the right-most sum is taken over all $q_{i}$ such that $\left.p \mid \sigma\left(q_{i}^{a_{i}}\right)\right)$. At most one of

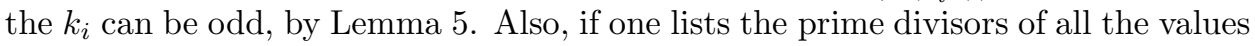
$\sigma\left(q^{k_{i}}\right)$ in the sum, there can appear no more than two distinct primes exceeding $10^{4}$, nor more than one prime between $10^{4}$ and $10^{6}$. Recalling how $A_{p}, B_{p}, C_{p}$ and $\lambda(p)$ are defined in Section 3 , the result follows.

We now state and prove

Lemma 8. Suppose for $p \in X$ and $d>\lambda(p)+1$, that $u v \mid \Phi_{d}(p)$, where $10^{4}<u<v$. Consider the following three statements:

1. $4 \mid o_{p}(Q)$, or $2 \mid o_{p}(Q)$ and $Q \equiv 3(\bmod 4)$.

2. Either $r$ is not $Q$-admissible for some $r \mid o_{p}(Q)$, or $Q \equiv 1(\bmod p)$ and $p$ is not $Q$-admissible.

3. There exists $t>10^{4}, t \neq u, t \neq v$, such that either $t \mid \Phi_{r}(Q)$ for some $r \mid o_{p}(Q)$, or $t \mid \Phi_{p}(Q)$ and $Q \equiv 1(\bmod p)$.

If, for both cases $Q=u$ and $Q=v$, any of these three statements is true, then $d \nmid v_{p}(N)+1$.

Proof. Suppose, for some $p \in X$, that $d \mid v_{p}(N)+1, d>\lambda(p)+1$, and $u v \mid \Phi_{d}(p)$, where $10^{4}<u<v$. Then $u=S$ and $v=P$, by (3) and (7). Thus, by Lemma 7 and equation (8), $p$ divides either $\sigma\left(S^{\beta}\right)$ or $\sigma\left(P^{\alpha}\right)$. Suppose $p \mid \sigma\left(S^{\beta}\right)$. Then statement 1 , as above with $Q=S$, is false by (4) and Lemma 5 Statements 2 and 3, with $Q=S$, are false by (4), (3), Definition 2, and (17). Similarly, if $p \mid \sigma\left(P^{\alpha}\right)$, then statements 1,2 , and 3 are false when $Q=P$.

We now wish to show that $d \nmid v_{p}(N)+1$ for certain cases where $\Phi_{d}(p)$ is divisible by exactly one prime exceeding $10^{4}$. Consider the set of 24 ordered pairs given by

$$
\begin{aligned}
Y=\{ & (3,13),(3,23),(5,7),(5,11),(5,13),(5,47),(7,13),(11,7), \\
& (11,9),(11,17),(11,19),(13,5),(13,7),(13,9),(19,19),(31,5), \\
& (31,7),(31,17),(61,7)(61,9),(61,23),(97,5),(97,11),(97,17)\} .
\end{aligned}
$$

Computation shows that each ordered pair is of the form $(p, d)$ for some $p \in X$ such that $\Phi_{d}(p)=J Q$, where $J \mid L$ and $Q>10^{4}$ (recall $L$ from (7)). Computation also shows that four important properties are shared by all $(p, d) \in Y$ :

1. Property A: For $r<100, r \neq p$, one of the following hold:

(a) $4 \mid o_{Q}(r)$ or $2 \mid o_{Q}(r)$ and $r \equiv 3(\bmod 4)$,

(b) $q t \mid o_{Q}(r), q t<o_{Q}(r), t=31,37$, or $t \geq 43$,

(c) $t$ is not $r$-admissible for some $t \mid o_{Q}(r)$.

2. Property B: Either 2 is not $Q$-admissible or $(p, d)$ is either $(13,9)$ or $(31,17)$. 
3. Property C: Either 3 is not $Q$-admissible or $(p, d)$ is one of $(3,23),(5,7)$, $(13,9),(31,17)$ or $(97,11)$.

Before stating Property D, we need the following result, which appears as Lemma 2 in [3]:

Lemma 9. Suppose $\Phi_{r}(p)=K q^{b}$, where $b \geq 1$ and $q \neq K$. Let $h=o_{p}(q)$ and $H=$ $o_{p}(K)$. Define $c$ to be the least nonnegative solution to the congruence $K^{h}-1 \equiv p x$ $\left(\bmod p^{2}\right)$. Then $p \| \Phi_{h}(q)$ if $H \neq c$.

A special case of Lemma 9] appears as Lemma 3 in [7]:

Lemma 10. If $\Phi_{r}(p)=q^{b}$ for some $b \geq 1$, and $h=o_{p}(q)$, then $p \| \Phi_{h}(q)$.

Then we have

4. Property D: For all $r$ such that $5 \leq r<100$, one of the following is true:

(a) 2 or 3 divides $o_{r}(Q)$.

(b) There exists $t$ which is not $Q$-admissible and is such that either $t \mid o_{r}(Q)$ or $Q \equiv 1(\bmod r)$ and $r$ is not $Q$-admissible.

(c) There exists $t \mid o_{r}(Q)$ such that $\Phi_{t}(Q)=K M($ or $Q \equiv 1(\bmod r)$ and $\left.\Phi_{r}(Q)=K M\right)$, where $q<100$ if $q \mid K, M>1, q>10^{4}$ if $q \mid M$, and writing $H=o_{Q}(K)$, we have $H \neq c$ if $K^{H}-1 \equiv c Q\left(\bmod Q^{2}\right)$.

We are now ready to state and prove

Lemma 11. If $(p, d) \in Y$, then $d \nmid v_{p}(N)+1$.

Proof. We use reductio ad absurdum. For, suppose $d \mid v_{p}(N)+1$ for some $(p, d) \in Y$. By our previous remarks, $\Phi_{d}(p)=J Q$, where $J \mid L$ and $Q>10^{4}$; furthermore, $(p, d)$ has Properties A, B, C and D. Thus $Q \mid N$ by (3); either $Q=S$ or $Q=P$ (in fact $Q=S$ if $Q<10^{6}$ ).

We first consider Property A. Let $r<100, r \neq p$, and suppose $r^{b} \| N$. If $4 \mid o_{r}(Q)$ or if $2 \mid o_{r}(Q)$ and $r \equiv 3(\bmod 4)$, then $Q \nmid \sigma\left(r^{b}\right)$ by (4) and Lemma 5 . If $q t \mid o_{Q}(r)$, $q t<o_{Q}(r)$, and $t=31,37$, or $t \geq 43$, then by (4), (3), and Lemmata (3) and (4), the assumption that $Q \mid \sigma\left(r^{b}\right)$ implies $N$ is divisible by two distinct primes, each exceeding 100, and, by Lemma 2 different from $Q$; this is impossible. Finally, if $t$ is not $r$-admissible for some $t \mid o_{Q}(r)$, then $Q \nmid \sigma\left(r^{b}\right)$ by (4) and Lemma 6. We conclude from Property A that $Q \nmid \sigma\left(r^{b}\right)$ if $r^{b} \| N$. Furthermore, $Q \nmid v_{p}(N)+1$ by (3) and Lemmata 3 and 4 . Thus by (4) we have $Q \| \sigma(L)$.

We next consider Property B. If 2 is not $Q$-admissible, then $Q \neq \pi$ by Lemmata 6] and 5] If we have $(p, d)=(13,9)$, then $Q=1609669$. Since $160967 \mid \Phi_{2}(Q)$, then $Q=\pi$ would imply $P=Q$ and $S=160967$, and hence $S \equiv 1(\bmod 13)$ while $o_{13}(P)=3$. Thus by Lemma 7 and 8 we have $13^{4} \mid \sigma\left(S^{\beta}\right) \sigma\left(p^{\alpha}\right)$. However, if $13^{2} \mid \sigma\left(S^{\beta}\right)$, then by (4), (3), and Lemmata 3 and 4, we have $Q \operatorname{Pr} \mid 2 N$ for some $r \equiv 1\left(\bmod 13^{2}\right)$, which is impossible. Thus $13^{3} \mid \sigma\left(P^{\alpha}\right)$. As $P^{3}-1 \equiv 143\left(\bmod 13^{2}\right)$, we have $13 \| \Phi_{3}(P)$ by (1), and thus it follows similarly that $13^{3} \nmid \sigma\left(P^{\alpha}\right)$. Finally, if we have $(p, d)=(31,17)$, then, as above, if $Q=\pi$, then $P \equiv 1(\bmod 31)$ and $o_{31}(S)=5$. By Lemma 7 and (8)), either $31^{6} \mid \sigma\left(P^{\alpha}\right)$ or $31^{6} \mid \sigma\left(S^{\beta}\right)$. Again, it is impossible to have $31^{6} \mid \sigma\left(P^{\alpha}\right)$; thus $31^{6} \mid \sigma\left(S^{\beta}\right)$. But $S^{5}-1 \equiv 930\left(\bmod 31^{2}\right)$; hence by (11) $31 \| \Phi_{5}(S)$. Thus by (14) $31^{4} \mid \beta+1$; again, this is impossible. We conclude from Property B that $Q \neq \pi$, and hence $v_{Q}(N)>1$.

We now consider Property C. By Lemma 6, $3 \nmid v_{Q}(N)+1$ if 3 is not $Q$-admissible. Otherwise, suppose $d \mid v_{p}(N)+1$, where $(p, d)$ is $(3,23),(5,7),(13,9),(31,17)$ or 
$(97,11)$. As previously noted, $\Phi_{d}(p)=J Q$, where $J \mid L$ and $Q>10^{4}$. Computation then shows that $\Phi_{3}(Q)=I R$, where $I \mid L$ and $R>Q$. Hence if $3 \mid v_{Q}(N)+1$, then $P=R$ and $S=Q$. In the case where $(p, d)=(3,23)$, we have $3^{6} \mid \sigma\left(S^{\beta}\right)$ or $3^{6} \mid \sigma\left(P^{\alpha}\right)$ by Lemma 7 and (8). As $o_{3}(S)=2$ and $S \neq \pi$, we have $3 \nmid \sigma\left(S^{\beta}\right)$ by (44) and Lemma 5 But $P \equiv 1(\bmod 3)$, and hence $3^{6} \nmid \sigma\left(P^{\alpha}\right)$ by (44), (3) and Lemmata 3 and 4 In the case where $(p, d)=(5,7)$, we have $5 \mid \sigma\left(S^{\beta}\right) \sigma\left(P^{\alpha}\right)$ by Lemma 7 and (88). But $S \equiv P \equiv 1(\bmod 5)$, where 5 is neither $S$-admissible nor $P$-admissible. Similarly, if $(p, d)=(13,9)$, then $13 \mid \sigma\left(S^{\beta}\right) \sigma\left(P^{\alpha}\right)$, but $o_{13}(P)=4$ (where 4 is not $P$-admissible), and $o_{13}(S)=6$ (by (4) and (3), $13 \mid \sigma\left(S^{\beta}\right)$ would imply $\Phi_{2}(S) \Phi_{3}(S) \Phi_{6}(S) \mid 2 N$, clearly a contradiction as the product involves three primes exceeding $\left.10^{4}\right)$. If $(p, d)=(31,17)$, then $31^{6} \mid \sigma\left(S^{\beta}\right)$ or $31^{6} \mid \sigma\left(P^{\alpha}\right)$. But $o_{31}(P)=30$, where 2 is not $P$-admissible, and $S \equiv 1(\bmod 31)$, implying by (4), (3) and Lemmata 3 and 4 that $31^{6} \nmid \sigma\left(S^{\beta}\right)$. Finally, in the case where $(p, d)=(97,11)$, we have $97 \mid \sigma\left(S^{\beta}\right) \sigma\left(P^{\alpha}\right)$ but $o_{97}(S)=16$ and $o_{97}(P)=32$. Thus we may conclude from Property C that $3 \nmid v_{Q}(N)+1$. Since also $2 \nmid v_{Q}(N)+1$, it follows from (8) that $Q^{2} \mid \sigma\left(P^{\alpha}\right)$ if $Q=S$, or $Q^{2} \mid \sigma\left(S^{\beta}\right)$ if $Q=P$.

Finally, we consider Property D. Write $\gamma=v_{Q}(N)$ and let $r<100$. If 2 or 3 divides $o_{r}(Q)$, then $r \nmid \sigma\left(Q^{\gamma}\right)$ by (4) and Properties B and C. Otherwise, we have two possibilities. The first is that $t$ is not $Q$-admissible for some $t \mid o_{r}(Q)$ (or $Q \equiv 1(\bmod r)$ and $r$ is not $Q$-admissible). In this case, $r \nmid \sigma\left(Q^{\gamma}\right)$ by (4) and Lemma 6. The second possibility is that either $\Phi_{t}(Q)=K M$ for some $t \mid o_{r}(Q)$, or $Q \equiv 1(\bmod r)$ and $\Phi_{r}(Q)=K M$, where $K \mid L$ and $q>10^{4}$ if $q \mid M$; furthermore, if $H=o_{Q}(K)$, we have $H \neq c$ if $K^{H}-1 \equiv c Q\left(\bmod Q^{2}\right)$. Now, if $t$ is not $Q$-admissible (or if $r$ is not $Q$-admissible in the case when $Q \equiv 1(\bmod r)$ ), then, as above, we have $r \nmid \sigma\left(Q^{\gamma}\right)$. Otherwise, $\Phi_{t}(Q)=K R^{b}$ for some $t \mid o_{r}(Q)$ (or, in the case when $\left.Q \equiv 1(\bmod r), \Phi_{r}(Q)=K R^{b}\right)$, where $b \geq 1$ and $R>10^{4}$. Suppose, then, that $r \mid \sigma\left(Q^{\gamma}\right)$. By (4) and (3), we have $S=Q$ and $P=R$ if $Q<R$, or we have $S=R$ and $P=Q$ if $Q>R$. Without loss of generality we may assume $S=Q$ and $P=R$. Let $h=o_{S}(P)$. Then Lemma 9 implies $S \| \Phi_{h}(P)$. Then $S \nmid v_{P}(N)+1$ if $S \mid \sigma\left(P^{\alpha}\right)$ by (4), (3), and Lemmata 3 and 4 Thus $v_{S}\left(\sigma\left(P^{\alpha}\right)\right) \leq 1$ by (44); this contradicts the conclusion at the end of the preceeding paragraph of this proof; namely, that $S^{2} \mid \sigma\left(P^{\alpha}\right)$. We conclude that $r \nmid \sigma\left(Q^{\gamma}\right)$ for all $r<100$ if $\gamma=v_{Q}(N)$.

Without loss of generality, let $S=Q$. Then, as $r \nmid \sigma\left(S^{\beta}\right)$ for all $r \mid L$, we have $\sigma\left(S^{\beta}\right)=P^{b}$ for some $b \geq 1$. By Lemma 10 we have $S \| \Phi_{h}(P)$ if $h=o_{S}(P)$. The same argument as in the preceeding paragraph tells us that $S \nmid \alpha+1$ and $v_{S}\left(\sigma\left(P^{\alpha}\right)\right) \leq 1$, which contradicts the fact, previously deduced from our hypothesis, that $S^{2} \mid \sigma\left(P^{\alpha}\right)$. This contradiction completes the proof of Lemma 11 .

In order to complete this section, we need one more auxiliary result; namely,

Lemma 12. If $5 \mid N$ and $5=\pi$, then $5 \| N$. Thus if $v_{5}(N)$ is odd, then $v_{5}(N)=1$.

Proof. Again, we use reductio ad absurdum. For, suppose $5=\pi$ and that $a>1$, $a=v_{5}(N)$. If $a=5$, then by (3) we have $5 \cdot \Phi_{2}(5) \Phi_{3}(5) \Phi_{6}(5) \mid 2 N$; this contradicts the well-known fact that no odd perfect number can be divisible by $3 \cdot 5 \cdot 7$. As 10 is not 5 -admissible, we have $a \neq 9$ by Lemma 6 . Thus $a \geq 13$. By Lemma 7 and (8) we have either $5^{6} \mid \sigma\left(S^{\beta}\right)$ or $5^{6} \mid \sigma\left(P^{\alpha}\right)$. If $5^{6} \mid \sigma\left(S^{\beta}\right)$, then $\beta+1$ is odd, as $S \neq \pi$. Hence by (44) $o_{5}(S)$ is odd; therefore $S \equiv 1(\bmod 5)$ and so $5^{6} \mid \beta+1$. But this is impossible by (3) and Lemmata (3) and (4), and the same argument precludes the possibility that $5^{6} \mid \sigma\left(P^{\alpha}\right)$. 
The reader may now note that Lemma 6 gives us by computation the following 67 ordered pairs $(p, d)$ for which $p \in X$ and $d \nmid v_{p}(N)+1:(3,7),(3,9),(3,11)$, $(3,15),(3,17),(3,19),(3,25),(3,31),(3,43),(3,47),(3,53),(3,67),(5,9),(5,10)$, $(5,15),(5,17),(5,19),(5,23),(5,25),(5,31),(5,37),(7,5),(7,7),(7,9),(7,11)$, $(7,19),(7,23),(7,31),(7,37),(11,5),(11,13),(11,23),(11,29),(11,31),(11,41)$, $(13,6),(13,11),(13,17),(13,23),(13,29),(19,3),(19,5),(19,7),(19,13),(19,23)$, $(19,29),(19,41),(31,3),(31,11),(31,13),(31,19),(31,23),(31,29),(61,5),(61,6)$, $(61,11),(61,17),(61,19),(61,29),(61,41),(97,3),(97,6),(97,7),(97,19),(97,23)$, $(97,29),(97,41)$.

Similarly, Lemma 8 gives the following 20 ordered pairs $(p, d)$ where $p \in X$ and $d \nmid v_{p}(N)+1:(3,29),(3,37)(3,41),(3,59),(3,61),(5,29),(5,41),(5,43)$, $(7,17),(7,29),(7,41),(11,11),(13,13),(13,19),(13,41),(19,11),(19,17),(31,41)$, $(61,13),(97,13)$.

Combining these remarks with the statements of Lemmata 11$]$ and 12, we summarize the results of this section by stating

Lemma 13. If $p \in X$ and $p \mid N$, let the notation $\left(p: j_{1}, \ldots, j_{k},[l]\right)$ mean that $v_{p}(N)=j_{1}, \ldots, j_{k}$ or $v_{p}(N) \geq l$. Then we have $(3: 2,4,[70]),(5: 1,2,4,[52])$, $(7: 2,[42]),(11: 2,[36]),(13: 1,2,[30]),(19:[30]),(31:[30]),(61: 1,2,[30])$, and $(97: 1,[30])$.

Furthermore, if $p \in X, p^{a} \| N$, and $a>4$, then there exists $r \mid a+1$ such that $q>100$ if $q \equiv 1(\bmod r)$.

\section{IMPROVED LOWER BOUNDS ON $S$ AND $P$}

By applying Lemma [13] we can dramatically increase the lower bounds given in Lemma 1 for $S$ and $P$. Indeed, in this section we show that $P>S>2^{32}$.

Montgomery [6] gives all solutions to $a^{q-1} \equiv 1\left(\bmod q^{2}\right)$ with $2 \leq a \leq 99$ and $q<2^{32}$. As a direct consequence of Montgomery's result, and by computation, we have

Lemma 14. If $p \in X, 10^{4}<q<2^{32}$, and $p^{q-1} \equiv 1\left(\bmod q^{2}\right)$, then $o_{q}(p)$ is composite.

For each $p \in X$, we may define $c_{p}$ and $d_{p}$ as follows:

$$
\begin{array}{rrrrrrrrrr}
p: & 3 & 5 & 7 & 11 & 13 & 19 & 31 & 61 & 97 \\
c_{p}: & 70 & 52 & 42 & 36 & 30 & 30 & 30 & 30 & 30 \\
d_{p}: & 21 & 14 & 12 & 10 & 9 & 9 & 7 & 6 & 5
\end{array}
$$

We next state the following result, which is verified by computation.

Lemma 15. For all $p \in X$, consider the congruences

$$
x^{p-1} \equiv 1\left(\bmod p^{d_{p}}\right) \quad \text { and } \quad x^{p-1} \equiv\left(1+p+\cdots+p^{d_{p}-1}\right)^{p-1}\left(\bmod p^{d_{p}}\right) .
$$

For any prime solution $q$ to either of these two congruences for any $p \in X$, we have $q>2^{32}$.

Now we may state and prove

Lemma 16. If $p \in X$ and $v_{p}(N)>4$, then $P>S>2^{32}$.

Proof. Suppose $p \in X$ and $v_{p}(N)>4$. Then $p^{c_{p}} \mid N$ by Lemma 13. Thus by Lemma [7 and (8) either $v_{p}\left(\sigma\left(S^{\beta}\right)\right)$ or $v_{p}\left(\sigma\left(P^{\alpha}\right)\right)$ is no less than the least integer which is 
greater than or equal to $\left(c_{p}-\lambda(p)\right) / 2$. If $p \mid \sigma\left(S^{\beta}\right)$, then $p^{4} \nmid \beta+1$ by (3) and Lemmata 3 and 4 Similarly, $p^{4} \nmid \alpha+1$ if $p \mid \sigma\left(P^{\alpha}\right)$. As

$$
d_{p} \leq\left\lceil\left(c_{p}-\lambda(p)\right) / 2\right\rceil-3,
$$

it follows from (4) that either $p^{d_{p}} \mid \Phi_{h}(S)$ or $p^{d_{p}} \mid \Phi_{h^{\prime}}(P)$, where $h=o_{p}(S)$ and $h^{\prime}=o_{p}(P)$ (recall the ceiling function, $\lceil x\rceil=$ the least integer not less than $x$ ).

If $p^{d_{p}} \mid \Phi_{h}(S)$, then $S^{h} \equiv 1\left(\bmod p^{d_{p}}\right)$ by (1). Since $h \mid p-1$, we have $S^{p-1} \equiv 1$ $\left(\bmod p^{d_{p}}\right)$, and so $S>2^{32}$ by Lemma 15 a fortiori $P>S>2^{32}$.

Otherwise, $p^{d_{p}} \mid \Phi_{h^{\prime}}(P)$; again, this implies $P^{p-1} \equiv\left(\bmod p^{d_{p}}\right)$. By Lemma 13 there exists $r \mid v_{p}(N)+1$ such that $q>100$ if $q \equiv 1(\bmod r)$. Thus $p \not \equiv 1(\bmod r)$. Thus by Lemma 2, for all $q \mid \Phi_{r}(p)$, we have $r=o_{q}(p)$, implying $q \equiv 1(\bmod r)$, or $q>100$. Thus by (3) and (7) we have

$$
\Phi_{r}(p)=1+p+\cdots+p^{r-1}=S^{b} P^{a},
$$

where $0 \leq a \leq \alpha, 0 \leq b \leq \beta$. Then $b>0$, as otherwise, by Lemma 10, $p \| \Phi_{h^{\prime}}(P)$, which is false.

Suppose $b>1$. Then $r=o_{S}(p)$ by Lemma 2. Hence, as $S^{2} \mid \Phi_{r}(p)$, we have $p^{S-1} \equiv 1\left(\bmod S^{2}\right)$. Thus $S>2^{32}$ by Lemma 14, for $o_{S}(p)$ is prime.

Otherwise, $b=1$. We may assume $a>0$, as otherwise $S=\Phi_{r}(p)>p^{r-1} \geq$ $3^{30}>2^{32}$ (recall that $r \geq 31$ by Lemma 13). Then

$$
1+p+\cdots+p^{r-1}=S P^{a} .
$$

Since $P^{p-1} \equiv 1\left(\bmod p^{d_{p}}\right)$ and $d_{p}<r$ (recall that $r \geq 31$, above), this gives us

$$
S^{p-1} \equiv\left(1+p+\cdots+p^{d_{p}-1}\right)^{p-1}\left(\bmod p^{d_{p}}\right),
$$

implying $S>2^{32}$ by Lemma 15 ,

We are now ready to prove

Lemma 17. $P>S>2^{32}$.

Proof. Either 7, 3 or 5 divides $N$, for otherwise, by (5) and (6), we have

$$
\sigma_{-1}(N) \leq \frac{10007}{10006} \cdot \frac{1000003}{1000002} \cdot \prod_{11 \leq p \leq 97} \frac{p}{p-1}<2 .
$$

Suppose $7 \mid N$. By Lemma 13, either $v_{7}(N)>4$, in which case $S>2^{32}$ by Lemma [16] or $7^{2} \| N$. In the latter case, as $19 \mid \Phi_{3}(7)$, we have $19 \mid N$ by (3), whence $S>2^{32}$ by Lemmata 13 and 16

Suppose $3 \mid N$. If $v_{3}(N)>4$, then $S>2^{32}$ by Lemma 16 Otherwise $v_{3}(N)=2$ or 4.

If $3^{2} \| N$, then $13 \| N$ as $13 \mid \Phi_{3}(3)$. Again, $S>2^{32}$ if $v_{13}(N)>4$; otherwise $v_{13}(N)=1$ or 2 . If $13 \| N$, then $7 \mid N$ (as $7 \mid \Phi_{2}(13)$ ); hence, as we have seen, $S>2^{32}$. If $13^{2} \| N$, then $61 \mid N$. Again, if $v_{61}(N)>4$, then $S>2^{32}$; otherwise $v_{61}(N)=1$ or 2. The former case gives us $31 \mid N$ (as $31 \mid \Phi_{2}(61)$ ), whence $S>2^{32}$ by Lemmata 13 and [16] If $61^{2} \| N$, then $97 \mid N$ (as $97 \mid \Phi_{3}(61)$ ); again, this implies $S>2^{32}$, as $v_{97}(N)>4$ or $97 \| N$, where $7 \mid \Phi_{2}(97)$.

If $3^{4} \| N$, then $11 \mid N$, as $11 \mid \Phi_{5}(3)$. If $v_{11}(N)>4$, then $S>2^{32}$; otherwise $11^{2} \| N$. As $7 \mid \Phi_{3}(11)$, this implies $7 \mid N$, and hence $S>2^{32}$.

Suppose $5 \mid N$. If $5 \| N$, then $3 \mid N$, and so $S>2^{32}$. If $v_{5}(N)>4$, then $S>2^{32}$ by Lemma [16. If $5^{2} \| N$, then $31 \mid N\left(\right.$ as $\left.31=\Phi_{3}(5)\right)$, but $v_{31}(N)>4$ and so $S>2^{32}$. 
Otherwise $5^{4} \| N$; thus $11 \mid N$ (as $\left.11 \mid \Phi_{5}(5)\right)$. As above, either $v_{11}(N)>4$ or $11^{2} \| N$; as we have already seen, either case implies $S>2^{32}$.

We may now restrict $v_{p}(N)$ for all $p<100$ (besides the elements of $X$ ). Thanks to the new bound on $S$ and $P$, we have the following immediate result:

Lemma 18. For the given primes $p$, along the given values $d$, we have $d \nmid v_{p}(N)+1$.

$\begin{array}{llllll}p & d & p & d & p & d \\ 17 & 3,4,5,7 & 43 & 2,3,5,7 & 71 & 2,3,5 \\ 23 & 2,5,7 & 47 & 2,5,7 & 73 & 3,4,5 \\ 29 & 5,7 & 53 & 3,4,5,7 & 79 & 2,5 \\ 37 & 4,5,7 & 59 & 2,3,5 & 83 & 2,3,5 \\ 41 & 3,4,5,7 & 67 & 2,5 & 89 & 3,5\end{array}$

Lemma 18 follows as a corollary to Lemma [17, since computation shows that either $d$ is not $p$-admissible or $\Phi_{d}(p)$ is divisible by a prime $q$ such that $10^{4}<q<2^{32}$. Since $S>2^{32}$, it follows that $d \nmid v_{p}(N)+1$.

We conclude this section with a few more auxiliary results. The proof of Theorem 1 will be completed in Section 6.

Lemma 19. $6 \nmid v_{37}(N)+1$.

Proof. Suppose otherwise. Then by (3) we have

$$
37 \cdot \Phi_{2}(37) \Phi_{3}(37) \Phi_{6}(37) \mid 2 N,
$$

or, equivalently

$$
3 \cdot 7 \cdot 19 \cdot 31 \cdot 37 \cdot 43 \cdot 67 \mid N
$$

If $3^{4} \mid N$ we have, by (5),

$$
\sigma_{-1}(N) \geq \sigma_{-1}\left(3^{4} \cdot 7^{2} \cdot 19 \cdot 31 \cdot 37 \cdot 43 \cdot 67\right)>2,
$$

a contradiction (recall that $7^{2} \mid N$, since $7 \neq \pi$ ). Otherwise $3^{2} \| N$, and hence $\Phi_{3}(3)=$ $13 \mid N$, giving

$$
\sigma_{-1}(N) \geq \sigma_{-1}\left(3^{2} \cdot 7^{2} \cdot 13 \cdot 19 \cdot 31 \cdot 37 \cdot 43 \cdot 67\right)>2 .
$$

Lemma 20. If $v_{3}(N)>4$ or $v_{5}(N)>4$, then $S$ or $P$ is the special prime.

Proof. If $v_{3}(N)>4$, then $v_{3}(N) \geq 70$ by Lemma [13. Thus, by Lemma 7 and (8), $3^{30} \mid \sigma\left(S^{\beta}\right)$ or $3^{30} \mid \sigma\left(P^{\alpha}\right)$. If $3^{30} \mid \sigma\left(S^{\beta}\right)$, then $3^{5} \nmid \beta+1$ by (3) and Lemmata 3 and 4 . Thus, by (4), $3^{26} \mid \Phi_{h}(S)$ (where $h$ denotes $o_{3}(S)$ ), and $h>1$. This implies $h=2$ and therefore, by Lemma 5, $S=\pi$. Similarly, $P=\pi$ if $3^{30} \mid \sigma\left(P^{\alpha}\right)$. The exact same argument shows that if $v_{5}(N)>4$, then $S$ or $P$ is the special prime.

Lemma 21. Neither 29 nor 89 is the special prime.

Proof. Suppose $29=\pi$. As $3 \cdot 5 \mid \Phi_{2}(29)$, we have $3 \cdot 5 \mid N$. Then $v_{3}(N)=2$ or 4 by Lemma 20; similarly $v_{5}(N)=2$ or 4 .

If $3^{4} \| N$, then $\Phi_{5}(3)=11^{2} \mid N$; thus by (5), $\sigma_{1}(N) \geq \sigma_{-1}\left(3^{4} \cdot 5^{2} \cdot 11^{2}\right)>2$. Similarly, $\Phi_{5}(5)=11 \cdot 71$; hence if $5^{4} \| N$, then $\sigma_{-1}(N) \geq \sigma_{-1}\left(3^{2} \cdot 5^{4} \cdot 22^{2} \cdot 71\right)>2$.

Thus $v_{3}(N)=v_{5}(N)=2$. As $\Phi_{3}(3)=13$ and $\Phi_{3}(5)=31$, we have $\sigma_{-1}(N) \geq$ $\sigma_{-1}\left(3^{2} \cdot 5^{2} \cdot 13^{2} \cdot 31^{2}\right)>2$. This contradiction shows that $29 \neq \pi$. The exact same argument shows that $89 \neq \pi$. 


\section{A COMPUTER SEARCH}

We are now ready to complete the proof of Theorem 1 We begin by summarizing the results of Lemmata 13, 18, 19] and 21] as follows:

Lemma 22. If $p<100$ and $p \mid N$, let the notation $\left(p: j_{1}, j_{2}, \ldots, j_{k},[l]\right)$ mean that $v_{p}(N)=j_{1}, \ldots, j_{k}$ or $v_{p}(N) \geq l$. Then we have $(3: 2,4,[70]),(5: 1,2,4[52])$, $(7: 2,[42]),(11: 2,[36]),(13: 1,2,[30]),(17: 1,[10]),(19:[30]),(23: 2,[8])$, $(29: 2,[8]),(31:[30]),(37: 1,2,[8]),(41: 1,[10]),(43:[10]),(47: 2,[8])$, $(53: 1,[10]),(59:[6]),(61: 1,2,[30]),(67: 2,[6]),(71:[6]),(73: 1,[6])$, $(79: 2,[6]),(83:[6]),(89:[6])$, and $(97: 1,[30])$. Furthermore, if $p^{a} \| N$ and $a>4$, then $p^{a}>2^{35}$.

For each $p<100$, let $a_{p}=v_{p}(N)$ and let

$$
L(p)= \begin{cases}\log \sigma_{-1}\left(p^{a_{p}}\right), & \text { if } 0 \leq a_{p} \leq 4, \\ \log (p /(p-1)), & \text { if } a_{p}>4\end{cases}
$$

Thus, as a consequence of (5),

$$
\log \sigma_{-1}\left(p^{a_{p}}\right) \leq L(p) .
$$

We now state

Lemma 23. $N$ must satisfy the inequality

$$
\left|\sum_{p<100} L(p)-\log 2\right|<2^{-31} .
$$

Proof. From (8) we have $\log \sigma_{-1}(L)+\log \sigma_{-1}\left(S^{\beta}\right)+\log \sigma_{-1}\left(P^{\alpha}\right)=\log 2$; that is

$$
\sum_{p<100} \log \sigma_{-1}\left(p^{a_{p}}\right)=\log 2-\log \sigma_{-1}\left(S^{\beta}\right)-\log \sigma_{-1}\left(P^{\alpha}\right) .
$$

Since by Lemma 17 $P>S>2^{32}$, we have by (5) and (6)

$$
\sigma_{-1}\left(P^{\alpha}\right)<\sigma_{-1}\left(S^{\beta}\right)<\frac{2^{32}+1}{2^{32}}=1+2^{-32} .
$$

Applying the inequality $\log (1+x)<x$ for $x>0$ to (11) gives $\log \sigma_{-1}\left(S^{\beta}\right)+$ $\log \sigma_{-1}\left(P^{\alpha}\right)<2^{-31}$, and hence, from (10),

$$
\log 2-2^{-31}<\sum_{p<100} \log \sigma_{-1}\left(p^{a_{p}}\right)
$$

Thus the left-hand inequality of the statement of the lemma follows from (9).

To prove the right-hand inequality, note that as a consequence of (10)

$$
\sum_{p<100} \log \sigma_{-1}\left(p^{a_{p}}\right)<\log 2
$$


Let $R(p)=L(p)-\log \sigma_{-1}\left(p^{a_{p}}\right)$. Then $R(p)=0$ if $a_{p} \leq 4$. Otherwise by Lemma 22 $p^{a_{p}}<2^{35}$, in which case

$$
\begin{aligned}
R(p) & =\log \frac{p}{p-1}-\log \frac{p^{a_{p}+1}-1}{p^{a_{p}}(p-1)} \\
& =\log \left(1+\frac{1}{p^{a_{p}+1}-1}\right) \\
& <\frac{1}{p^{a_{p}+1}-1}<\frac{1}{2^{36}}
\end{aligned}
$$

so that $R(p)<1 /\left(2^{36}\right)$. Hence

$$
\sum_{p<100} R(p)<\frac{24}{2^{36}}<2^{-31} .
$$

Thus, by (12) and (13),

$$
\begin{aligned}
\sum_{p<100} L(p) & =\sum_{p<100} \log \sigma_{-1}\left(p^{a_{p}}\right)+\sum_{p<100} R(p) \\
& <\log 2+2^{-31},
\end{aligned}
$$

and so the lemma is proved.

At this stage, it was desired to conduct a computer search for all odd positive integers which satisfy Lemmata 22 and 23 To make the search more manageable, more restrictions were sought. For example, it is well known that if $N$ is an odd perfect number then $3 \cdot 5 \cdot 7 \nmid N$. In fact, our hypotheses on $N$, along with elementary arguments, enable us to deduce the following additional restrictions: $3 \cdot 5 \cdot 11 \nmid N$, if $5 \| N$, then $3^{2} \| N$; if $3^{4} \| N$, then $5 \nmid N$; if $3^{2} \| N$, then $v_{5}(N) \notin\{2,4\}$; and if $7^{2} \| N$, then $3^{2} \| N$ or $v_{3}(N)>4$.

These additional restrictions were incorporated in the computer search, making it more feasible. It was conducted on an IBM-486PC, using a UBASIC software package, as were all computations for this paper. The search was conducted for all odd positive integers with no prime divisors exceeding 100, which satisfy Lemmata 22 and 23. along with the additional restrictions discussed above. No such integers were found.

We conclude that Theorem 1 holds for all odd perfect numbers.

\section{Some CONCLUding REMARKS}

Thanks to Lemma 1 and Theorem 1 we now have $\mathcal{P}\left(K, 10^{8-2 K}\right)$ for $K=1$, 2 , and 3 (and vacuously for $K=4$ ). These lower bounds obtained for the first, second, and third largest prime divisors of an odd perfect number could be extended by applying the same techniques in the papers in which these results appeared, provided one has sufficient computer capability, energy, and patience.

I would like to express my thanks to Peter Hagis Jr., who took the time to proofread an earlier draft of this paper. My gratitude extends as well to the referee, whose suggestions concerning this paper (as well as [5]) were most helpful.

\section{REFERENCES}

1. L. Adleman, C. Pomerance, and R. Rumely, On distinguishing prime numbers from composite numbers, Ann. of Math. 117 (1973), 173-206. MR 84e:10008 
2. G. Cohen and P. Hagis, Every odd perfect number has a prime factor which exceeds $10^{6}$, Research Report R93-5, University of Technology, Sydney, (1993); Math. Comp. 67 (1998), 1323-1330. MR 98k:11002

3. P. Hagis, On the second largest prime divisor of an odd perfect number, Analytic Number Theory-Lecture Notes in Mathematics, Springer-Verlag, Berlin and New York, 899, 1981, 245-263. MR 83i:10004

4. P. Hagis, The third largest prime factor of an odd multiperfect number exceeds 100, Bull. Malaysian Math. Soc. 9 (1986), 43-49. MR 89b:11008

5. D. Iannucci, The second largest prime divisor of an odd perfect number exceeds ten thousand, to appear in Math. Comp.

6. P. Montgomery, New solutions of $a^{p-1} \equiv 1\left(\bmod p^{2}\right)$, Math. Comp. 61 (1993), 361-363. MR 94d:11003

7. C. Pomerance, The second largest prime factor of an odd perfect number, Math. Comp. 29 (1975), 914-921. MR 51:8018

University of the Virgin Islands, 2 John Brewers Bay, St. Thomas, Vi 00802

E-mail address: diannuc@uvi.edu 